MARINA BARGUIL MACÊDO

\title{
PAPEL PROTETOR DA QUINASE ATIVADA POR ADENOSINA MONOFOSFATO (AMPK) NA PROGRESSÃO E SEVERIDADE DA NEFRITE TUBULOINTERSTICIAL EXPERIMENTAL
}

Dissertação apresentada ao Programa de PósGraduação em Imunologia do Instituto de Ciências Biomédicas da Universidade de São Paulo para obtenção do título de Mestre em Ciências

Área de concentração: Imunologia

Orientador: Prof. Dr. Niels Olsen Saraiva Câmara

Versão original

SÃO PAULO

2017 


\section{RESUMO}

Macêdo MB. Papel protetor da quinase ativada por adenosina monofosfato (AMPK) na progressão e severidade da nefrite tubulointersticial experimental. [Dissertação (Mestrado em Imunologia)]. São Paulo: Instituto de Ciências Biomédicas, Universidade de São Paulo; 2017.

A quinase ativada por adenosina monofosfato (AMPK) é um sensor e regulador metabólico, cujo efeito sobre a polarização de macrófagos (MØ) vem sendo investigado como potencial alvo terapêutico. Objetivou-se investigar o papel da ativação dessa molécula pela metformina (Met) na doença renal crônica (DRC) experimental, tendo por hipótese que ela exerceria um impacto positivo sobre a progressão da doença, ao levar os M $\varnothing$ para um fenótipo menos próinflamatório. Para tal, inicialmente induziu-se nefrite tubulointersticial (NTI) crônica em camundongos C57BL/6, através de ração suplementada com adenina $0,25 \%$ por 10 dias. Concomitantemente, os animais foram tratados com Met $200 \mathrm{mg} / \mathrm{kg} / \mathrm{dia}$ ou salina, por gavagem. Intencionando comprovar que o efeito da Met sobre os MØ era dependente de AMPK, experimento idêntico foi realizado em camundongos LyzM-cre AMPK ${ }^{\text {flox/flox }}$. Com o intuito de identificar outras células relevantes para a fisiopatologia da lesão, e passíveis de alteração metabólica pela AMPK, administrou-se clodronato lipossomal $50 \mathrm{mg} / \mathrm{kg}$, uma droga depletora de M $\varnothing$, em camundongos C57BL/6 com NTI, e associou-se a gavagem com Met. Trabalhou-se ainda com camundongos CD4-cre AMPK ${ }^{\text {flox/flox }}$. Realizou-se experimento in vitro com células tubulares epiteliais renais murinas (CTERM, linhagem MM55.K), em que se avaliou o efeito do estímulo com Met $20 \mathrm{mM}$ sobre a transição epitélio-mesenquimal (TEM). Os camundongos C57BL/6 tratados com Met apresentaram menor deterioração da função renal, ao que se associou uma redução do número de granulomas no interstício renal; uma maior frequência de $\mathrm{M} \varnothing \mathrm{M} 1$, em detrimento dos M2; e uma redução da expressão da citocina pró-fibrótica TGF- $\beta$. Os camundongos LyzM-cre $\mathrm{AMPK}^{-/-}$não diferiram dos LyzMcre $\mathrm{AMPK}^{+/+}$quanto à intensidade da lesão, o que se justifica por a molécula já se encontrar menos expressa no contexto da NTI. Por outro lado, ao se estimular a fosforilação da quinase com Met, os LyzM-cre $\mathrm{AMPK}^{+/+}$evoluíram melhor do que os não tratados, o mesmo não se verificando nos LyzM-cre $\mathrm{AMPK}^{-/}$, o que sugere que a ação da Met nos MØ é de fato dependente de AMPK. Apesar disso, a Met ainda foi capaz de limitar o grau de fibrose nos rins dos LyzM-cre $\mathrm{AMPK}^{-/}$. O experimento com o Clo apontou para a existência de outros alvos celulares da Met, uma vez que os animais tratados com ambas as drogas se mostraram significativamente melhores do que os que receberam apenas uma delas. Os camundongos CD4-cre $\mathrm{AMPK}^{-/-}$e CD4-cre $\mathrm{AMPK}^{+/+}$manifestaram a doença com igual gravidade. Não houve diferenças no infiltrado linfocitário dos linfonodos renais entre os grupos controle e com NTI, o que insinua que os linfócitos T CD4, nesse modelo de DRC, desempenham papel pouco relevante para o estabelecimento da lesão. As CTERM apresentaram aumento da fosforilação da AMPK e ativação da via glicolítica após o estímulo com Met, ao que se correlacionou maior captação de glicose e expressão do transportador Glut-2. Houve menos TEM nas CTERM que receberam Met. Assim, conclui-se que, na NTI experimental, a ativação da AMPK reduz acentuadamente a gravidade da doença, ao modular os MØ para um fenótipo menos pró-fibrótico, e ao tornar as CTERM resistentes à TEM.

Palavras chave: Quinase Ativada por Adenosina Monofosfato (AMPK). Imunometabolismo. Macrófagos. Metformina. Doença renal crônica. 


\begin{abstract}
Macêdo MB. Protective role of adenosine monophosphate activated kinase (AMPK) on the progression and severity of experimental tubulointerstitial nephritis. [Dissertação (Mestrado em Imunologia)]. São Paulo: Instituto de Ciências Biomédicas, Universidade de São Paulo; 2017.
\end{abstract}

Adenosine monophosphate activated kinase (AMPK) is an energy sensor and a master regulator of metabolism, whose role on macrophage (MØ) polarization has been proposed as a potential therapeutic target. We aimed to investigate the role of AMPK activation by metformin (Met) on chronic kidney disease, hypothesizing that it could have a positive impact on disease progression, mainly by leading $\mathrm{M} \varnothing$ to a less inflammatory phenotype. We induced tubulointerstitial nephritis (TIN) on C57BL/6 mice by feeding of adenine-enriched $(0.25 \%)$ diet for 10 days. Mice were gavaged daily with either saline or Met $200 \mathrm{mg} / \mathrm{kg}$. In order to certify that Met was acting through an AMPK-dependent pathway on MØ, the same model was tested in LyzM-cre AMPK ${ }^{\text {flox/flox }}$ mice. With the intent of identifying other cells meaningful to TIN pathogenesis, and prone to the beneficial interference of AMPK on their metabolism, we administered liposome-encapsulated clodronate $50 \mathrm{mg} / \mathrm{kg}$ to C57BL/6 mice with TIN, and additionally treated them with Met. We also worked with CD4-cre $\mathrm{AMPK}^{\text {flox/flox }}$ mice. We performed an in vitro experiment on a lineage of murine renal tubular epithelial cells (RTEC, MM55.K) to assess the effect of Met $20 \mathrm{mM}$ on epithelial to mesenchymal transition (EMT). Met-treated C57BL/6 mice presented a less marked deterioration of renal function, to which it was associated a reduction of the number of granulomata on renal interstitium; a greater frequency of infiltrating M1 MØ; and a reduced expression of pro-fibrotic cytokine TGF- $\beta$. Severity of renal injury did not differ between LyzM-cre $\mathrm{AMPK}^{-/-}$and $\mathrm{AMPK}^{+/+}$mice, what can be explained by AMPK being downregulated on TIN. On the other hand, upregulation promoted by Met resulted in improvement of LyzM-cre $\mathrm{AMPK}^{+/+}$, but not $\mathrm{AMPK}^{-/}$mice, thus suggesting that the effect of Met on M $\varnothing$ is indeed dependent of AMPK. Nevertheless, Met was able to reduce renal fibrosis on LyzM-cre AMPK ${ }^{-/}$mice. The experiment with Clo pointed to other cellular targets of Met, since mice treated with both drugs faired even better than those treated with each one in separate. CD4-cre $\mathrm{AMPK}^{+/+}$and $\mathrm{AMPK}^{-/-}$mice presented the same intensity of injury, and there was no difference on the lymphocytic infiltrate of renal lymph nodes from control and diseased mice, indicating that CD4 T cells, on this model, do not present a prominent role. Met-stimulated RTEC expressed higher levels of phosphorylated AMPK, and exhibited activation of glycolysis, to which correlated increase of glucose uptake and enhanced expression of the transporter Glut-2. EMT was impaired on Met-treated RTEC. We therefore conclude that, in experimental TIN, activation of AMPK sensibly reduces disease severity both by modulating MØ to a less fibrotic phenotype, and turning RTEC resistant to EMT.

Keywords: Adenosine monophosphate activated kinase (AMPK). Immunometabolism. Macrophages. Metformin. Chronic kidney disease. 


\subsection{A doença - considerações clínico-epidemiológicas}

Definida como uma anormalidade funcional ou estrutural do rim que persiste por mais de três meses, com implicações para a saúde do indivíduo, a doença renal crônica (DRC) vem, de forma crescente, angariando atenção das comunidades médica e acadêmica ao se estabelecer como um problema de saúde pública [2].

Estima-se que entre 11 e $13 \%$ da população mundial apresente DRC. Esta cifra é particularmente alarmante ao se constatar que pode representar uma subestimativa, sendo o diagnóstico feito comumente de forma tardia, uma vez que os pacientes não costumam apresentar queixas a não ser em estágios avançados, quando a terapia renal substitutiva já se torna imperativa como pilar da proposta terapêutica [3].

Associada ao fato de ser uma condição indolente e assintomática em seus estágios iniciais, outra razão para o subdiagnóstico da perda da capacidade de filtração dos rins é a falta de uma sistematização visando a seu rastreamento. De fato, como ocorre com outras doenças crônico-degenerativas com que ela compartilha fatores de risco, tais como hipertensão arterial sistêmica e diabetes mellitus, a DRC entra no rol de doenças preveníveis. Assim, na atualidade, a investigação ativa de seus fatores causais, aliada à intervenção precoce, é a principal estratégia de que se dispõe para tentar reduzir sua prevalência [4].

Apesar de se ter conhecimento de uma abordagem custo-efetiva para conter seu alastramento, opções terapêuticas para os mais de 200 milhões de pacientes que já convivem com a doença são escassas, de modo que, anualmente, cerca de 850 mil indivíduos vão a óbito pela DRC, que assim se configura como a $12^{\mathrm{a}}$ causa de mortalidade no mundo [5-7].

Sabe-se que, a despeito de qual seja a causa subjacente à deterioração da função renal, a fisiopatologia da DRC envolve um estado persistente de inflamação local e sistêmica que resulta na perda do parênquima renal, que gradativamente vai sendo substituído por fibrose. Logo, a identificação de alvos celulares e moleculares que permitam a interrupção de uma cascata inflamatória que tende a se amplificar e se retro-alimentar, levando à inexorável perda da função e à desestruturação anatômica dos rins, representa o cerne das pesquisas atuais que buscam sobrepujar o problema [8-9].

Contudo, a elaboração de novos compostos que possam vir a se tornar drogas no combate à progressão da DRC é um processo lento, longo e laborioso, sendo premente o surgimento de alternativas que possam, ao menos a médio prazo, constar como opção terapêutica viável e acessível [10]. 
Nesse contexto, o entendimento das mudanças metabólicas que ocorrem nas células do sistema imune, as principais responsáveis pelos danos estruturais que se estabelecem durante a progressão da doença, representou um avanço na busca de possíveis soluções terapêuticas para a DRC. Diante da perspectiva de modulação de vias imunometabólicas, uma molécula em especial tem despertado o interesse da comunidade científica por já ter um modulador largamente utilizado na prática clínica - essa molécula é a quinase ativada por adenosina monofosfato (AMPK), e seu modulador de emprego corrente é a metformina (Met) [11].

\subsection{A molécula - considerações químico-estruturais e funcionalidades no sistema imune}

A AMPK é um heterotrímero composto por três subunidades, $\alpha, \beta$ e $\gamma$, pertencente à família das serina/treonina quinases. Expressa em todos os organismos eucarióticos, trata-se de uma quinase conservada evolutivamente com função de sensor energético, capacitando a célula a responder a desafios energéticos. A exposição a ambientes pobres em glicose ou o aumento da concentração intracelular de AMP desencadeiam a fosforilação da treonina 172 da subunidade $\alpha$, com função catalítica, estimulando, portanto, a ativação da quinase $[12,13]$.

Várias são as vias metabólicas que convergem para ou interagem com a AMPK. Enquanto algumas têm o propósito de transmitir estímulos tróficos vindos do extracelular, mediando a comunicação entre a célula e o meio em que ela se insere (a exemplo dos receptores hormonais de adiponectina e leptina), outras sinalizam para alterações no próprio intracelular (como a disfunção mitocondrial causando a elevação da razão AMP/ADP, um forte gatilho para deflagrar a ação da AMPK) [14].

Muito ainda se desconhece sobre quais sejam as moléculas intermediárias entre a AMPK e os receptores localizados na membrana celular; sabe-se, porém, que as três principais quinases envolvidas na fosforilação da treonina 172 são a quinase dependente de cálcio-calmodulina (CAMKK $\beta)$, a quinase hepática B1 (LKB1), e a quinase ativada pelo fator transformador do crescimento $\beta$ (TAK1) [15].

Tem-se demonstrado que variadas situações fisiológicas e patológicas são capazes de deflagrar a ativação da AMPK, por exemplo, a contração muscular, a hipóxia, a inflamação e a sepse [13]. Todas essas situações convergem quanto ao fato de representarem um stress metabólico, requisitando da célula um alto consumo energético.

A AMPK concorre para o estabelecimento de um balanço energético favorável agindo em duas frentes: ao regular positivamente as vias catabólicas que produzem trifosfato de adenosina (ATP), e negativamente as vias anabólicas que consomem ATP [16]. Desta forma, 
em condições de escassez de glicose, a AMPK atua tanto na redução do gasto energético, suprimindo a tradução de ácido ribonucleico mensageiro (RNAm) de citocinas, quanto na ativação de vias metabólicas alternativas, como o ciclo dos ácidos tricarboxílicos, que utiliza o aminoácido não essencial glutamina como substrato básico [17,18].

Figura 1 - Modelo da via de sinalização da AMPK.

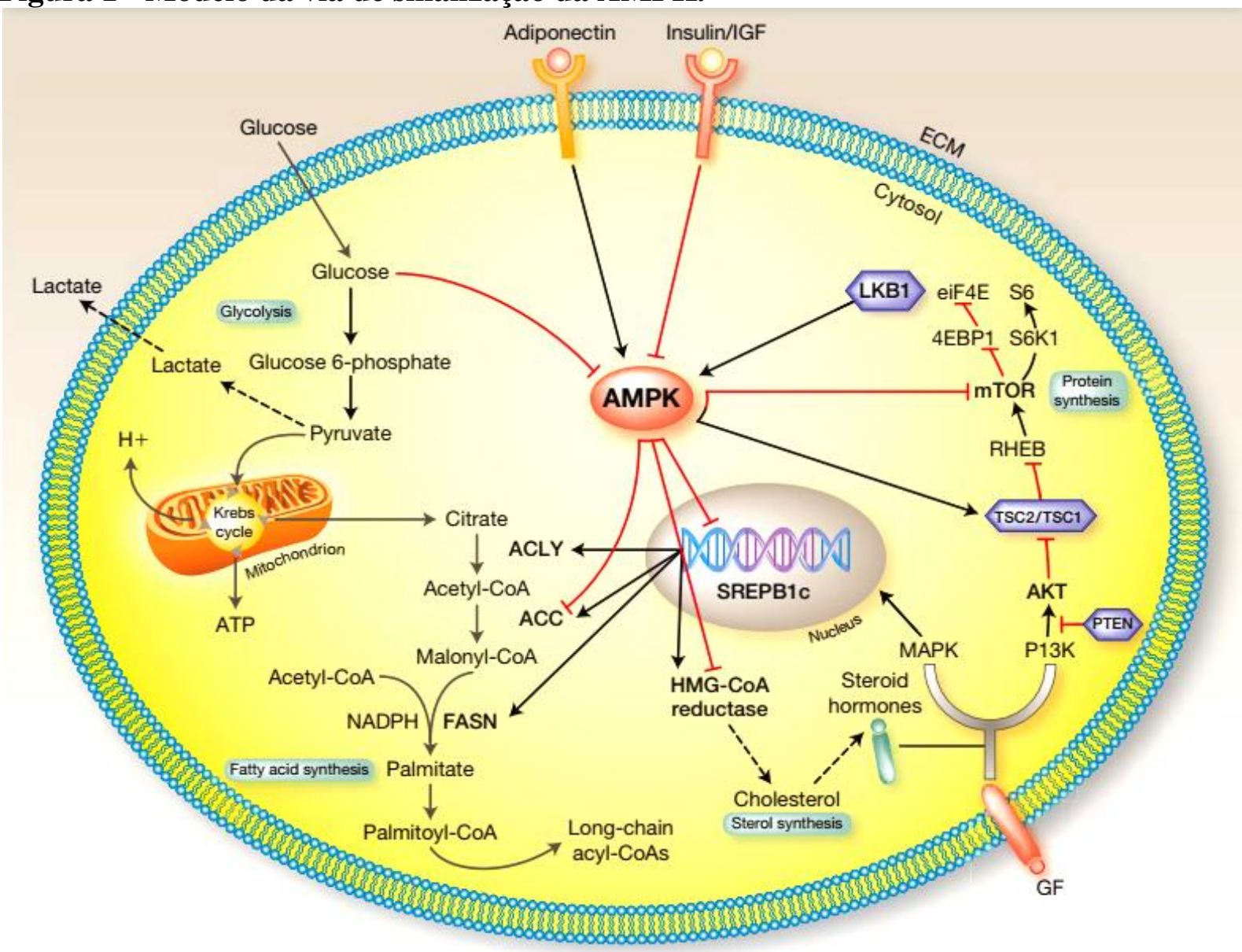

A AMPK age em concerto com outras quinases no sentido de promover redução do consumo e aumento da produção de energia pela célula. Para tal, ela reprime enzimas envolvidas em vias anabólicas, seja através da inibição de sua transcrição, ao interagir com a proteína de ligação do elemento regulador de esterol (SREBP1c), seja através da fosforilação de enzimas relacionadas à etapa limitante de reações, tais como a hidróxi-metilglutaril-CoA redutase (HMG-CoA redutase) e a acetil-CoA carboxilase (ACC), que controlam a síntese de lipídios e ácidos graxos. Além de seus efeitos negativos sobre a lipidogênese, a AMPK também suprime a síntese proteica ao inibir direta e indiretamente (via ativação do complexo da esclerose tuberosa - TSC) o complexo do alvo molecular da rapamicina (mTOR). Paralelamente, a AMPK eleva a síntese de trifosfato de adenosina (ATP) ao estimular a glicólise e o ciclo do ácido tricarboxílico (ciclo de Krebs). Fonte: Zadra et al. (2010) [19].

O estudo in vivo realizado por Blagih et al. (2015) demonstrou que linfócitos T (LT) nos quais se induziu deficiência da isoforma AMPKal apresentam maior apoptose quando submetidos a ambientes adversos em termos de oferta nutricional [12]. Tais ambientes, apesar de elaborados artificialmente no estudo em questão, são, em verdade, a realidade com a qual os LT devem confrontar durante sua transição de nä̈ve, quando habitam órgãos linfoides em 
condições otimizadas de oferta nutricional, para efetores, quando passam a ocupar sítios em que a competição por nutrientes, oxigênio e fatores de crescimento é a regra, conforme sugeriu estudo de Pearce et al. (2013) [20].

Além de atuar como moduladora do metabolismo celular no processo de diferenciação dos LT, a AMPK também exerce papel central na mudança do padrão de comportamento dos macrófagos. Seu envolvimento na polarização dos macrófagos do fenótipo de M1 para M2, já foi demonstrado em diversos estudos. Isso porque ela parece ser particularmente relevante para as células cuja principal fonte energética advém da oxidação dos ácidos graxos, como é o caso dos macrófagos M2 [21,22].

Ao promover a biogênese mitocondrial, através da estimulação do coativador do receptor ativado pela proliferação de peroxissomos $\gamma$ (PPAR $\gamma)$, e ao elevar captação de ácidos graxos pela mitocôndria, através da estimulação da carnitina palmitoiltransferase $1 \alpha(\mathrm{Cpt} 1 \alpha)$, a AMPK maximiza a síntese de citocinas antiinflamatórias pelos macrófagos M2, como a IL10 [23]. Não apenas isso: a AMPK também está inserida em várias vias de sinalização implicadas na regulação negativa da inflamação, agindo, por exemplo, através da inibição do fator nuclear kappa B (NF-kB) e da ativação da serina/treonina quinase AKT [24].

\subsection{0 agonista - considerações mecanísticas e terapêuticas}

A modulação positiva da AMPK em uma série de modelos experimentais de lesão renal tanto aguda quanto crônica abre uma perspectiva de interferir na sua via de sinalização para reduzir a progressão e a severidade de diversas nefropatias. Essa perspectiva é particularmente promissora uma vez que se dispõe de uma droga amplamente comercializada, e de segurança comprovada em seres humanos, que tem como mecanismo de ação a estimulação da fosforilação da AMPK - trata-se da Met, atualmente indicada para controle glicêmico no diabetes tipo II $[25,26]$.

Por extensão, dispõe-se de inúmeras publicações acerca dos benefícios da Met na nefropatia diabética, os quais foram recentemente sumarizados em uma revisão de Ravindran et al. (2016), mas o seu potencial de aplicabilidade terapêutico é muito mais amplo. Isso porque todos os insultos renais, independentemente de sua natureza, tendem a evoluir para o estabelecimento de fibrose tecidual [27].

Em um estudo de Lu et al. (2015), evidenciou-se que a Met é capaz de inibir a produção de colágeno mediada pelo fator de transformação do crescimento beta (TGF- $\beta$ ) em 
fibroblastos renais, de modo que ela agiria, dessa forma, interrompendo a via final comum a diversos mecanismos de injúria renal [28].

Corroborando as evidências in vitro, Cavaglieri et al. (2015) demonstraram em um modelo de obstrução ureteral unilateral que camundongos tratados apresentam menor infiltrado inflamatório no rim obstruído, além de menor expressão de citocinas inflamatórias e marcadores de fibrose [29].

\subsection{As células - considerações fisiopatológicas}

Os macrófagos representam uma das populações celulares mais comumente identificadas em espécimes oriundos de tecido renal que sofreu agressão, seja ela imunomediada ou não. Tem-se atribuído a eles tanto efeitos patogênicos, ao propiciar, em seu fenótipo M1, um ambiente pró-inflamatório nas fases iniciais da doença renal, e ao estimular, em seu fenótipo M2, a fibrogênese em fases mais tardias, quanto efeitos benéficos, ao promover o clareamento de imunocomplexos e estimular a reparação do tecido lesado [30].

Apesar dessa abrangência de ações que poderiam, a princípio, serem caracterizadas como díspares, sabe-se que a interação dos macrófagos com as células glomerulares e tubulares epiteliais renais é mediada por citocinas e fatores de crescimento responsáveis por regular os efeitos dos macrófagos na DRC, podendo estes, na dependência daqueles, assumir um papel positivo ou negativo sobre o processo lesão-reparação [31].

De modo que, irrespectivamente da natureza inicial do insulto, ao sistema imune é atribuído o papel central em manter ou sustar a injúria tecidual, estando claro que a predominância de uma subpopulação de macrófagos, M1 ou M2, tem implicações decisivas sobre a evolução da lesão renal [30,31].

Isto abre possíveis janelas de oportunidades terapêuticas quanto à interferência nesta transição entre os polos M1 e M2, impedindo que a subpopulação implicada no ciclo repetitivo inflamação-fibrose perpetue o processo. $\mathrm{Na}$ DRC, ao mesmo tempo em que o fenótipo M2 está envolvido na regeneração da membrana tubular basal e na secreção de fatores antiinflamatórios, também a ele se imputa o recrutamento de miofibroblastos e o estímulo à síntese de colágeno. Assim, o próprio controle fino dos papéis exercidos por um mesmo fenótipo é campo a ser desbravado no interesse de se encontrar novos alvos terapêuticos [32].

Em contrapartida, a presença de LT CD4+ no infiltrado celular de um rim cronicamente lesado se relaciona com a diminuição da função renal e com a extensão da 
fibrose. A fibrose intersticial e atrofia tubular são similarmente achados não específicos compatíveis com uma multiplicidade de causas, incluindo lesão renal isquêmica, hipertensão e toxicidade crônica por medicamentos [33].

A fibrose intersticial é caracterizada por um acúmulo de tecido conjuntivo, que inclui colágenos tipo I e III, fibronectina e vários proteoglicanos, levando a uma desorganização na arquitetura do rim [34]. O seu aparecimento é uma sequela de inflamação ou lesão, durante as quais fatores humorais são liberados por células infiltrantes ou residentes, que estimulam o parênquima renal a aumentar a produção de componentes da matriz extracelular, como o colágeno, sendo a intensidade de fibrose intersticial o processo mais fortemente relacionado com a deterioração da função do enxerto renal no pós-transplante [35]. Especificamente na rejeição crônica do enxerto mediada por células, ocorre ativação de LT, fibrose intersticial e atrofia tubular, sem evidência de alguma etiologia específica [36].

\section{Figura 2 - Representação esquemática dos mecanismos celulares envolvidos na progressão da doença renal crônica.}

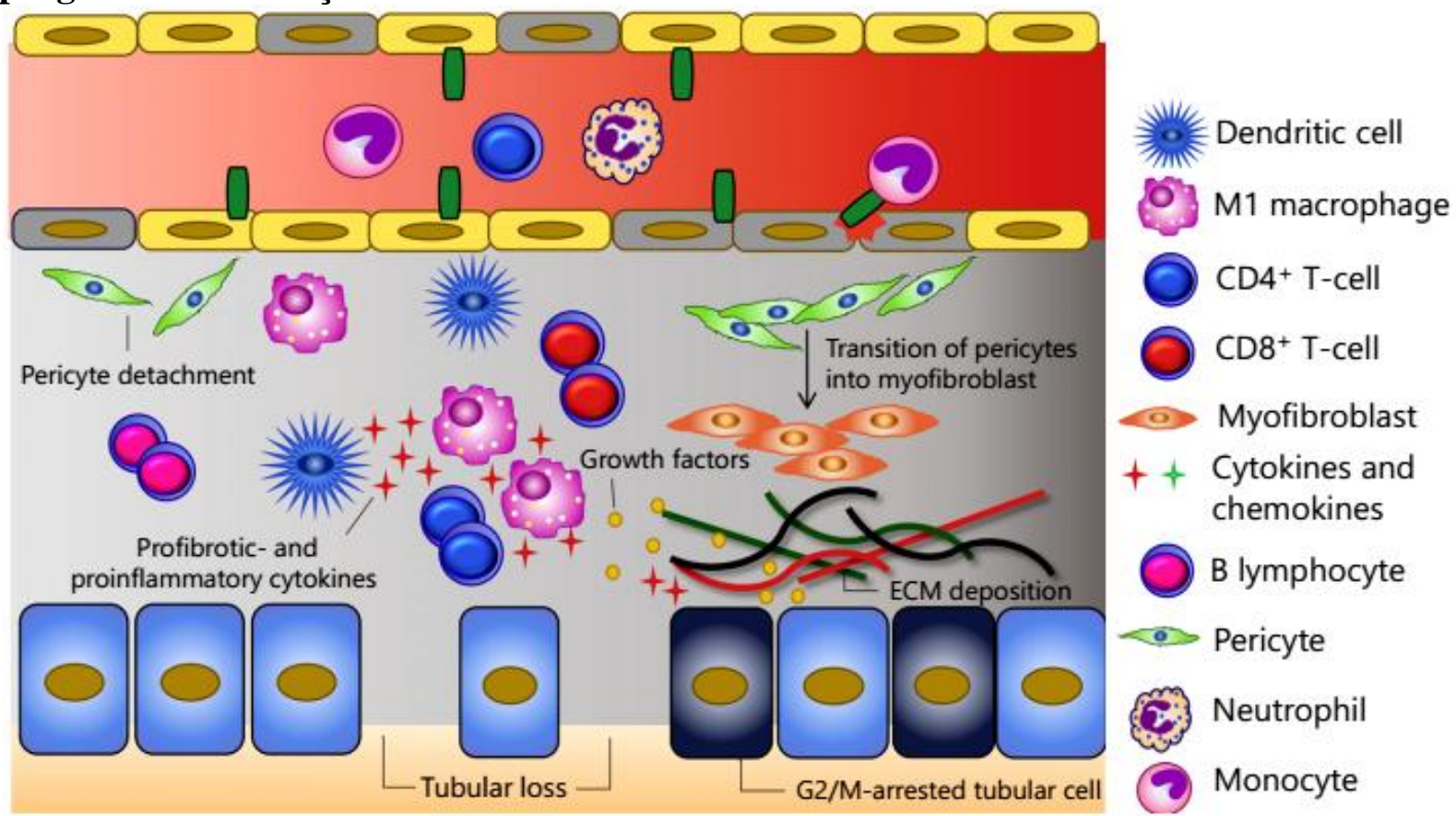

Independentemente do fator causal da lesão, o processo que leva à cronificação da doença renal envolve uma interação viciosa entre as células do sistema imune e as células tubulares epiteliais: macrófagos e linfócitos T secretam citocinas pró-inflamatórias que, por sua vez, mantêm o influxo constante de células infiltrantes no tecido, amplificando o sinal; ao mesmo tempo, fatores de crescimento, a exemplo do fator transformador do crescimento beta (TGF- $\beta$ ), são liberados e induzem transição epitélio-mesenquimal, convertendo pericitos e miofibroblastos produtores de matriz extracelular (ECM), o que agrava ainda mais a destruição da arquitetura tubular, que acaba por ser substituída por fibrose. Fonte: Lee et al (2017) [37].

Os LT CD4+ sintetizam e secretam fator transformador do crescimento beta (TGF- $\beta$ ), molécula com importante função fibrogênica. O TGF- $\beta$ estimula a produção de moléculas como colágeno e fibronectina pelos fibroblastos. Estudos mostraram que, durante a DRC em 
camundongos, há uma massiva infiltração de LT CD4+ e secreção de citocinas tais como IFN- $\gamma$ e IL-1 $\beta$ [38].

Ainda, a transferência para camundongos saudáveis de LT CD4+ provenientes de camundongos submetidos à isquemia e reperfusão renal em uma fase tardia, com significante presença de fibrose e inflamação, rapidamente induziu aumento de albuminúria nos receptores [39]. Esses dados indicam que uma alteração no metabolismo dessas células é capaz de induzir alterações significativas nas funções renais.

\subsection{O modelo - considerações fisio e histopatológicas}

Em mamíferos, a adenina (Ad) é um produto endógeno da via da poliamina e é captada pela adenina-fosforibosiltransferase (APRT). Quando a APRT funcional está ausente, a Ad passa a ser um substrato significante para a xantina-desidrogenase (XDH), que a oxida em 2,8-diidroxiadenina (DHA) [40].

Como a 2,8-DHA é extremamente insolúvel, sua excreção pelo rim pode levar à formação de cálculos urinários. Os cristais de 2,8-DHA exercem efeitos tóxicos diretos nas células tubulares e intersticiais renais, eventualmente levando à falência renal de estágio final $[41,42]$.

Os mecanismos envolvidos na toxicidade tubular não são bem compreendidos. Estudos experimentais com camundongos deficientes de APRT apresentam nefrolitíase com extensa dilatação tubular, inflamação, necrose e fibrose [43]. Além disso, a Ad é uma base purínica, sendo, portanto, precursora do ácido úrico (AU) na via da xantina oxidase. O AU, por seu turno, é reconhecidamente um importante deflagrador de estresse oxidativo, podendo, por si só, resultar em DRC [44].

Do ponto de vista histopatológico, secções de tecido renal de ratos ou camundongos com lesão renal mediada por Ad revelam predominantemente uma desestruturação da arquitetura tubular, sendo o glomérulo relativamente poupado. Assim, o uso de ração enriquecida com Ad para induzir DRC experimental constitui um interessante modelo de nefrite tubulointersticial (NTI) crônica [45].

A NTI, em humanos, é definida microscopicamente por fibrose intersticial e atrofia tubular com variado infiltrado inflamatório de linfócitos, macrófagos e outras células, desde que a lesão intersticial esteja fora de proporção para qualquer doença glomerular. Fibrose periglomerular é comum e algumas obsolescências glomerulares podem ser encontradas nas áreas afetadas [46]. 


\subsection{Justificativa}

O acúmulo de evidências sobre a importância do papel da AMPK no metabolismo celular, cuja função não é restrita ao equilíbrio energético, mas também se relaciona à resposta inflamatória e à fibrose tecidual, vem estimulando o estudo desta molécula como alvo terapêutico potencial em uma série de doenças [47,48].

Por sua vez, a Met, um agonista reconhecido da AMPK, é capaz de reduzir a expressão de TGF- $\beta$ em fibroblastos renais de camundongos, o que, por sua vez, resulta em menor produção de colágeno por essas células [28,49].

A relevância de tal constatação é significante uma vez que se sabe que a fibrose e a produção desregulada de colágeno constituem o ponto crucial no remodelamento tecidual verificado na DRC. A possibilidade de se intervir sobre essa etapa do processo patológico, para o qual no momento não se dispõe ainda de uma droga modificadora da história natural da doença, abre um campo de oportunidades para o desenvolvimento de fármacos voltados para a modulação do AMPK e de outros componentes que com ele interagem na cascata [50].

Isso nos motivou a elaborar o presente estudo, que visa avaliar os efeitos da AMPK sobre o curso da DRC, presumindo-se que sua ativação module os dois principais tipos celulares presumivelmente envolvidos na lesão, os macrófagos e linfócitos T CD4+, a apresentar um fenótipo menos pró-inflamatório. 
O presente estudo permite concluir que:

(i) A Met apresenta papel protetor na NTI crônica induzida por Ad;

(ii) A preservação da função renal está associada ao aumento da fosforilação da AMPK no tecido renal;

(iii) A principal célula modulada pela Met no contexto da lesão renal por Ad é o MØ;

(iv) O tratamento com Met resulta em redução do número de granulomas envolvendo cristais de adenina nos rins, se associando a maior preservação arquitetural do tecido;

(v) O marcador de miofibroblasto $\alpha$ SMA é fortemente expresso nos rins doentes, e a Met leva à normalização para níveis verificados em rins saudáveis;

(vi) O colágeno I, principal componente da matriz extracelular na fibrose intersticial que caracteriza a DRC, tem sua expressão diminuída pela Met;

(vii) Os camundongos com deleção seletiva de AMPK nos MØ não apresentam diferenças na função renal quando comparados aos AMPK selvagem, o que pode ser explicado por a fração ativa da quinase se encontrar menos expresa no contexto da doença;

(viii) A administração de Met aos camundongos LyzM-cre $\mathrm{AMPK}^{-/-}$não exerce influência sobre a gravidade da doença, enquanto que esta é amenizada de forma apreciável naqueles LyzM-cre $\mathrm{AMPK}^{+/+}$, comprovando que a modulação do MØ proporcionada pela droga tem um mecanismo dependente de AMPK (Figura 54);

(ix) Ainda assim, os camundongos LyzM-cre $\mathrm{AMPK}^{-/-}$expressam menos $\alpha \mathrm{SMA}$ nos rins, apontando que a ativação de AMPK em outras células também contribui para os efeitos benéficos da Met;

(x) A associação de CEL, uma droga depletora de MØ, com a Met proporciona melhora clínica ainda mais acentuada, ratificando a existência de outros alvos celulares do agonista de AMPK;

(xi) O infiltrado linfocitário no rim e no linfonodo renal dos grupos Ad não apresenta diferenças comparativamente aos grupos controles em camundongos CD4-cre AMPK ${ }^{\text {flox/flox }}$, excluindo os LT CD4 como células relevantes no estabelecimento da lesão;

(xii) O tratamento de CTERM com Metresulta em aumento da fosforilação da AMPK e em resistência à TEM induzida por TGF- $\beta$; e

(xiii) A resistência verificada se associa a uma mudança do perfil metabólico dessas células, que passam a captar mais glicose, expressar mais Glut2, e ativar a via glicolítica.

O mecanismo molecular e celular proposto para a ação da Met na NTI induzida por Ad encontra-se sumarizado na Figura 55. 
Figura 54 - Esquema representativo do mecanismo de ação da metformina na nefrite tubulointersticial induzida por adenina em camundongos LyzM-cre AMPK ${ }^{\text {flox/flox }}$.



Fonte: autoria própria. 


\section{Figura 55 - Sumário gráfico.}

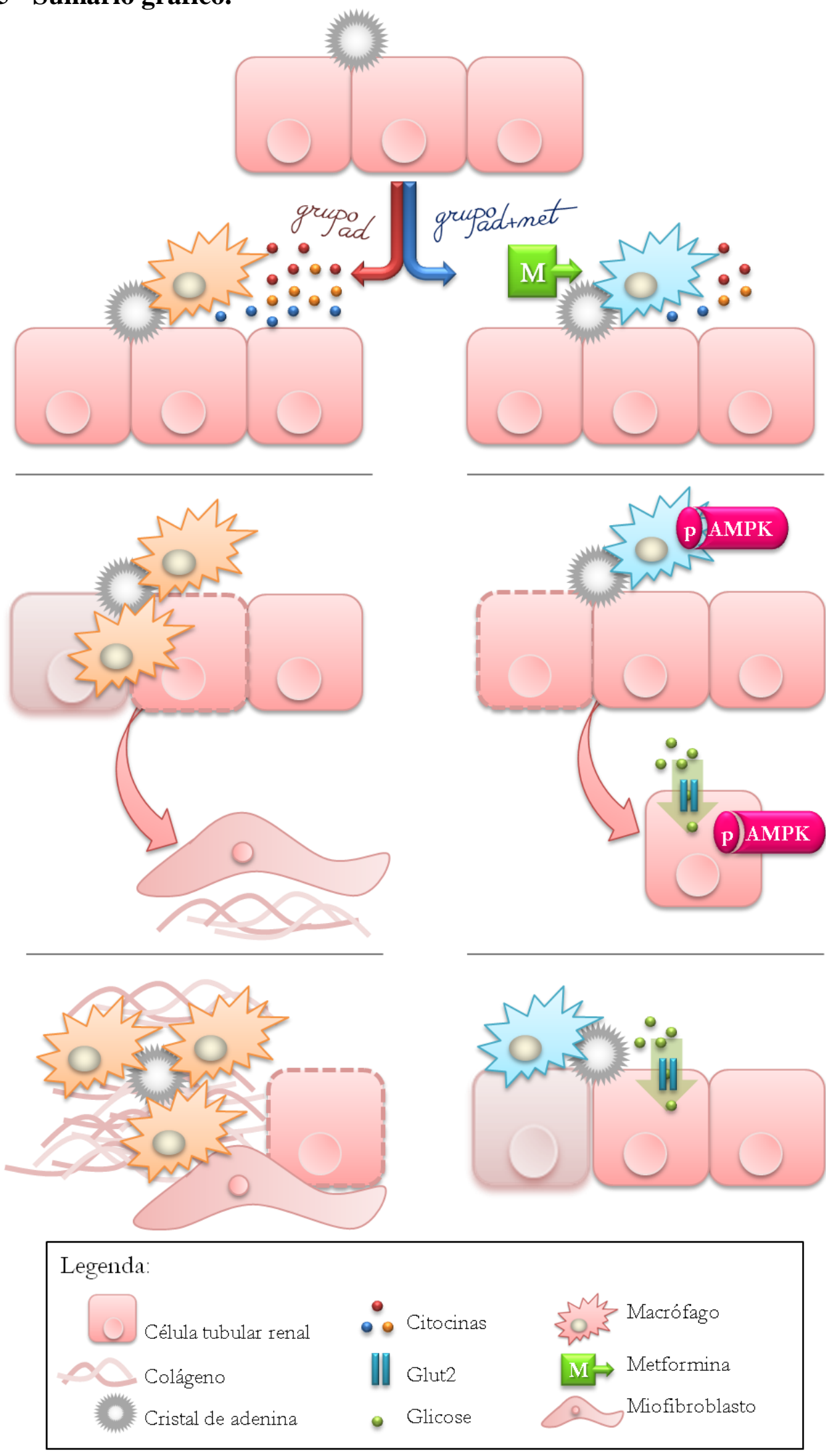

A adenina é convertida em cristais insolúveis de 2,8-diidroxiadenina pela xantina oxidase, os quais se depositam no interstício renal. Nos camundongos selvagens não tratados (esquema à esquerda), ocorre ativação dos macrófagos residentes e recrutamento de monócitos infiltrantes que, juntos produzem citocinas próinflamatórias, como IL-6 e TNF- $\alpha$. A lesão inflamatória, juntamente da obstrução mecânica promovida pelos cristais, leva à destruição do epitélio tubular. Concomitantemente, células epiteliais, sob o estímulo de citocinas 
pró-fibróticas, como o TGF- $\beta$, sofrem transição epitélio-mesenquimal, se transformando em miofibroblastos produtores de matriz extracelular. Assim, progressivamente, o parênquima é substituído por tecido fibrótico. $\mathrm{O}$ tratamento com metformina (esquema à direita) leva à fosforilação de AMPK em macrófagos e células tubulares epiteliais. Nestas, ela induz aumento da expressão do transportador de glicose Glut2, ao que se associa maior captação de glicose e ativação da via glicolítica, alterações metabólicas que tornam as células resistentes à TEM. Naqueles, a ativação da AMPK inibe a inflamação, resultando em menor formação de granulomas e, consequentemente, menor deterioração da arquitetura tubular. Os rins tratados com metformina, desta forma, apresentam menos marcadores de fibrose ( $\alpha$ SMA e colágeno) e têm sua função preservada. Fonte: autoria própria. 


\section{REFERÊNCIAS ${ }^{*}$}

1. Sinclair L. Arrowsmith. New York: Signet Classics, 1925.

2. Kidney Disease: Improving Global Outcomes (KDIGO) CKD Work Group. KDIGO 2012 Clinical Practice Guideline for the Evaluation and Management of Chronic Kidney Disease. Kidney inter., Suppl. 2013; 3: 1-150.

3. Hill NR, Fatoba ST, Oke JL et al. Global Prevalence of Chronic Kidney Disease - A Systematic Review and Meta-Analysis. PLoS One. 2016; 11(7): e0158765.

4. Fishbane S, Hazzan AD, Halinski C, Mathew AT. Challenges and opportunities in late-stage chronic kidney disease. Clin Kidney J. 2015; 8 (1): 54-60.

5. Schieppati A, Remuzzi G. Chronic renal diseases as a public health problem: epidemiology, social, and economic implications. Kidney Int Suppl. 2005 Sep;(98):S7-S10.

6. Schoolwerth AC, Engelgau MM, Rufo KH, et al. Chronic Kidney Disease: A Public Health Problem That Needs a Public Health Action Plan. Preventing Chronic Disease. 2006;3(2):A57.

7. Perico N, Remuzzi G. Chronic kidney disease: a research and public health priority. Nephrol Dial Transplant. 2012 Oct;27 Suppl 3:iii19-26.

8. Silverstein DM. Inflammation in chronic kidney disease: role in the progression of renal and cardiovascular disease. Pediatr Nephrol. 2009 Aug;24(8):1445-52.

9. Akchurin OM, Kaskel F. Update on inflammation in chronic kidney disease. Blood Purif. 2015;39(1-3):84-92.

10. Ojo A. Addressing the Global Burden of Chronic Kidney Disease Through Clinical and Translational Research. Trans Am Clin Climatol Assoc. 2014; 125: 229-246.

11. Norata GD, Caligiuri G, Chavakis T et al. The Cellular and Molecular Basis of Translational Immunometabolism. Immunity. 2015 Sep 15;43(3):421-34.

12. Blagih J, Coulombe F, Vincent EE, et al. The Energy Sensor AMPK Regulates T Cell Metabolic Adaptation and Effector Responses In Vivo. Immunity. 2015 Jan; 42:41-54.

13. Andris F, Leo O. AMPK in Lymphocyte Metabolism and Function. International Reviews of Immunology. 2015; 34(1):67-81.

14. Dasgupta B, Chippa RR. Evolving Lessons on the Complex Role of AMPK in Normal Physiology and Cancer. Trends Pharmacol Sci. 2016 Mar;37(3):192-206.

15. Fullerton MD1, Steinberg GR. "Presenting" an adaptive role for AMPK. J Leukoc Biol. 2013 Dec;94(6):1099-101. doi: 10.1189/jlb.0613335.

*De acordo com Estilo Vancouver. 
16. Mayer A, Denanglaire S, Viollet B, et al. AMP-activated protein kinase regulates lymphocyte responses to metabolic stress but is largely dispensable for immune cell development and function. Eur. J. Immunol. 2008; 38:948-956.

17. Cham CM, Driessens G, O'Keefe JP, et al. Glucose deprivation inhibits multiple key gene expression events and effector functions in CD8+ T cells. Eur. J. Immunol. 2008; 38:24382450.

18. Le A, Lane AN, Hamaker M, et al. Glucose-independent glutamine metabolism via TCA cycling for proliferation and survival in B cells. Cell Metab. 2012; 15:110-121.

19. Zadra G, Priolo C, Patnaik A, Loda M. New strategies in prostate cancer: targeting lipogenic pathways and the energy sensor AMPK. Clin Cancer Res. 2010 Jul 1;16(13):3322-8. doi: 10.1158/1078-0432.CCR-09-1955.

20. Pearce EL, Poffenberger MC, Chang CH, et al. Fueling Immunity: Insights into Metabolism and Lymphocyte Function. Science. 2013 Oct; 342(6155):1242-454.

21. Mounier R, Théret M, Arnold L et al. AMPKa1 Regulates Macrophage Skewing at the Time of Resolution of Inflammation during Skeletal Muscle Regeneration. Cell Metabolism. $2013 ; 18: 251-264$.

22. Weng SY, Schuppan D. AMPK regulates macrophage polarization in adipose tissue inflammation and NASH. Journal of Hepatology. 2013; 58:619-621.

23. Kelly B, O’Neill, LAJ. Metabolic reprogramming in macrophages and dendritic cells in innate immunity. Cell Research. 2015; 25:771-784.

24. Sag D, Carling D, Stout RD, Suttles J. AMP-activated protein kinase promotes macrophage polarization to an anti-inflammatory functional phenotype. J Immunol. 2008 December 15; 181(12): 8633-8641.

25. Zhou G, Myers R, Li Y et al. Role of AMP-activated protein kinase in mechanism of metformin action. J Clin Invest. 2001 Oct 15; 108(8): 1167-1174.

26. Viollet B, Guigas B, Garcia NS et al. Cellular and molecular mechanisms of metformin: an overview. Clin Sci (Lond). 2012 Mar; 122(6): 253-270.

27. Ravindran S, Kuruvilla V, Wilbur K, Munusamy S. Nephroprotective Effects of Metformin in Diabetic Nephropathy. J Cell Physiol. 2017 Apr; 232(4):731-742.

28. Lu J, Shi J, Li M, Gui B, Fu R et al. Activation of AMPK by metformin inhibits TGF- $\beta$ induced collagen production in mouse renal fibroblasts. Life Sci. 2015 Apr 15;127:59-65.

29. Cavaglieri RC, Day RT, Feliers D, Abboud HE. Metformin prevents renal interstitial fibrosis in mice with unilateral ureteral obstruction. Mol Cell Endocrinol. 2015 Sep 5;412:116-22. doi: 10.1016/j.mce.2015.06.006. 
30. Duffield JS. Macrophages and Kidney Disease: Macrophages and Immunological Inflammation of the kidney. Semin Nephrol. 2010 May; 30(3): 234-254. doi:10.1016/j.semnephrol.2010.03.003.

31. Tian S, Chen SY. Macrophage polarization in kidney diseases. Macrophage 2015; 2: e679. doi: 10.14800

32. Kinsey GR. Macrophage Dynamics in AKI to CKD Progression. Journal of the American Society of Nephrology 2014; 25: 209-11.

33. Racusen LC, Solez K, Colvin RB, et al. The Banff 97 working classification of renal allograft pathology. Kidney Int 1999; 55(2):713-23.

34. Muller GA, Schettler V, Muller CA, Strutz F. Prevention of progression of renal fibrosis: how far are we? Kidney Int Suppl 1996; 54:S75-82.

35. Sund S, Reisaeter AV, Fauchald $P$ et al. Living donor kidney transplants: a biopsy study 1 year after transplantation, compared with baseline changes and correlation to kidney function at 1 and 3 years. Nephrol Dial Transplant 1999; 14(10):2445-54.

36. Solez K, Colvin RB, Racusen LC et al. Banff '05 Meeting Report: differential diagnosis of chronic allograft injury and elimination of chronic allograft nephropathy ('CAN'). Am J Transplant 2007; 7(3):518-26.

37. Lee SA, Noel S, Sadasivam M, Hamad ARA, Rabb H. Role of Immune Cells in Acute Kidney Injury and Repair. Nephron. 2017 Jun 10. doi: 10.1159/000477181.

38. Burne-Taney MJ, Yokota N, Rabb H. Persistent renal and extrarenal immune changes after severe ischemic injury. Kidney Int 2005; 67:1002-1009.

39. Burne-Taney MJ, Liu M, Ascon D et al. Transfer of lymphocytes from mice with renal ischemia can induce albuminuria in naive mice: a possible mechanism linking early injury and progressive renal disease? Am J Physiol Renal Physiol 2006; 291:F981-F986.

40. Stockelman MG, Lorenz JN et al. Chronic renal failure in a mouse model of human adenine phosphoribosyltransferase deficiency. Am J Physiol, 1998; 275(1 Pt 2): F154-63.

41. Bouzidi H, Lacour B et al. 2,8-dihydroxyadenine nephrolithiasis: from diagnosis to therapy. Ann Biol Clin, 2007; 65(6): 585-92.

42. Daudon M, Traxer O et al. Lithogenesis. Prog Urol, 2008; 18(12): 815-27.

43. Engle SJ, Stockelman MG, et al. Adenine phosphoribosyltransferase-deficient mice develop 2,8-dihydroxyadenine nephrolithiasis. Proc Natl Acad Sci 1996; 93(11): 5307-12.

44. Diwan V, Mistry A, Gobe G, Brown L. Adenine-induced chronic kidney and cardiovascular damage in rats. J Pharmacol Toxicol Methods. 2013 Sep-Oct;68(2):197-207.

45. Jia $\mathrm{T}$, Olauson $\mathrm{H}$, Lindberg $\mathrm{K}$ et al. A novel model of adenine-induced tubulointerstitial nephropathy in mice. BMC Nephrol. 2013 May 30;14:116. 
46. John R, Herzenberg AM. Renal toxicity of therapeutic drugs. J Clin Pathol. 2009; 62(6): 505-15.

47. Galic S, Fullerton MD, Schertzer JD et al. Hematopoietic AMPK $\beta 1$ reduces mouse adipose tissue macrophage inflammation and insulin resistance in obesity. J Clin Invest. 2011 Dec;121(12):4903-15.

48. Lim JY, Oh MA, Kim WH, et al. AMP-activated protein kinase inhibits TGF-beta-induced fibrogenic responses of hepatic stellate cells by targeting transcriptional coactivator p300. J. Cell. Physiol. 2012; 227:1081-1089.

49. Lee JH, Kim JH, Kim JS et al. AMP-activated protein kinase inhibits TGF-, angiotensin II-, aldosterone-, high glucose-, and albumin-induced epithelial-mesenchymal transition. Am J Physiol Renal Physiol 304: F686-F697, 2013.

50. Boor P, Šebeková K, Ostendorf T, Floege J. Treatment targets in renal fibrosis. Nephrol. Dial. Transplant. 2007; 22 (12): 3391-3407.

51. Weissser SB, van Rooijen N, Sly LM. Depletion and Reconstitution of Macrophages in Mice. J Vis Exp. 2012 Aug 1;(66):4105.

52. van Rooijen N, Hendrikx E. Liposomes for Specific Depletion of Macrophages from Organs and Tissues. Methods Mol Biol. 2010; 605:189-203.

53. Pautz A, Art J, Hahn S, Nowag S, Voss C, Kleinert H. Regulation of the expression of inducible nitric oxide synthase. Nitric Oxide. 2010; 23(2): 75-93. doi: 10.1016/j.niox.2010.04.007.

54. Poljakovic M, Karpman D, Svanborg C, Persson K. Human renal epithelial cells express iNOS in response to cytokines but not bacteria. Kidney Int. 2002 Feb;61(2):444-55.

55. Kipari T, Cailhier J-F, Ferenbach D, et al. Nitric Oxide Is an Important Mediator of Renal Tubular Epithelial Cell Death in Vitro and in Murine Experimental Hydronephrosis. The American Journal of Pathology. 2006;169(2):388-399. doi:10.2353/ajpath.2006.050964.

56. Seidal T, Balaton AJ, Battifora H. Interpretation and quantification of immunostains. Am J Surg Pathol. 2001 Sep;25(9):1204-7.

57. Walker RA. Quantification of immunohistochemistry - issues concerning methods, utility and semiquantitative assessment I. Histopathology. 2006 Oct;49(4):406-10.

58. Taylor CR, Levenson RM. Quantification of immunohistochemistry - issues concerning methods, utility and semiquantitative assessment II. Histopathology. 2006 Oct;49(4):411-24.

59. Jensen EC. Quantitative Analysis of Histological Staining and Fluorescence Using ImageJ. Anat Rec (Hoboken). 2013 Mar;296(3):378-81.

60. Ina K, Kitamura H, Tatsukawa S, Fujikura Y. Significance of $\alpha$-SMA in myofibroblasts emerging in renal tubulointerstitial fibrosis. Histol Histopathol. 2011; 26: 855-866. 
61. Strutz F, Zeisberg M. Renal Fibroblasts and Myofibroblasts in Chronic Kidney Disease. J Am Soc Nephrol. 2006; 17: 2992-2998.

62. Vogel B, Siebert H, Hofmann U, Frantz S. Determination of collagen content within picrosirius red stained paraffin-embedded tissue sections using fluorescence microscopy. MethodsX. 2015 Feb 21;2:124-34.

63. Marks J, Carvou NJ, Debnam ES, Srai SK, Unwin RJ. Diabetes increases facilitative glucose uptake and GLUT2 expression at the rat proximal tubule brush border membrane. J Physiol. 2003;553(Pt 1):137-145.

64. Qaseem A, Barry MJ, Humphrey LL, Forciea MA. Oral Pharmacologic Treatment of Type 2 Diabetes Mellitus: A Clinical Practice Guideline Update From the American College of Physicians. Ann Intern Med. 2017 Jan 03; 166(4): 279-290.

65. Lipska KJ, Bailey CJ, Inzucchi SE. Use of Metformin in the Setting of Mild-to-Moderate Renal Insufficiency. Diabetes Care 2011 Jun; 34(6): 1431-1437.

66. Banerjee P, Surendran H, Chowdhury DR, Prabhakar K, Pal R. Metformin mediated reversal of epithelial to mesenchymal transition is triggered by epigenetic changes in E-cadherin promoter. J Mol Med. 2016; 94:1397-1409.

67. Mak RH, Ikizler AT, Kovesdy CP et al. Wasting in chronic kidney disease. J Cachexia Sarcopenia Muscle. 2011 Mar; 2(1): 9-25.

68. Wang XH, Mitch WE. Mechanisms of muscle wasting in chronic kidney disease. Nat Rev Nephrol. 2014 Sep; 10(9): 504-516.

69. Turner PV, Brabb T, Pekow C, Vasbinder MA. Administration of Substances to Laboratory Animals: Routes of Administration and Factors to Consider. Journal of the American Association for Laboratory Animal Science : JAALAS. 2011;50(5):600-613.

70. Lopez-Giacoman S, Madero M. Biomarkers in chronic kidney disease, from kidney function to kidney damage. World Journal of Nephrology. 2015;4(1):57-73. doi:10.5527/wjn.v4.i1.57.

71. Fernández I, Peña A, Teso ND, Pérez V, Rodríguez-Cuesta J. Clinical Biochemistry Parameters in C57BL/6J Mice after Blood Collection from the Submandibular Vein and Retroorbital Plexus. Journal of the American Association for Laboratory Animal Science : JAALAS. 2010;49(2):202-206.

72. Gowda S, Desai PB, Kulkarni SS et al. Markers of renal function tests. N Am J Med Sci. 2010 Apr; 2(4): 170-173.

73. Waanders F, van Timmeren MM, Stegeman CA, Bakker SJ, van Goor H. Kidney injury molecule-1 in renal disease. J Pathol. 2010 Jan;220(1):7-16.

74. Han WK, Bailly V, Abichandani R, Thadhani R, Bonventre JV. Kidney Injury Molecule-1 (KIM-1): a novel biomarker for human renal proximal tubule injury. Kidney Int. 2002 Jul;62(1):237-44. 
75. Bonventre JV. Kidney Injury Molecule-1 (KIM-1): a specific and sensitive biomarker of kidney injury. Scand J Clin Lab Invest Suppl. 2008;241:78-83.

76. Fong D, Ullah MM, Lal JG et al. Renal cellular hypoxia in adenine-induced chronic kidney disease. Clin Exp Pharmacol Physiol. 2016;43: 896-905. doi:10.1111/1440-1681.12621

77. Hayashi S, Oe Y, Fushima $\mathrm{T}$ et al. Protease-activated receptor 2 exacerbates adenineinduced renal tubulointerstitial injury in mice. Biochem Biophys Res Commun. 2017;483(1):547-552.doi: 10.1016/j.bbrc.2016

78. Nogueira A, Pires MJ, Oliveira PA. Pathophysiological Mechanisms of Renal Fibrosis: A Review of Animal Models and Therapeutic Strategies. In Vivo. 2017; 31(1): 1-22. doi:10.21873/invivo.11019.

79. O’Neill WC. Renal Relevant Radiology: Use of Ultrasound in Kidney Disease and Nephrology Procedures. Clinical Journal of the American Society of Nephrology : CJASN. 2014;9(2):373-381. doi:10.2215/CJN.03170313.

80. Yang X, Ding H, Qin Z, et al. Metformin Prevents Renal Stone Formation through an Antioxidant Mechanism In Vitro and In Vivo. Oxidative Medicine and Cellular Longevity. 2016;2016:4156075. doi:10.1155/2016/4156075.

81. Tamura M, Aizawa R, Hori M, Ozaki H. Progressive renal dysfunction and macrophage infiltration in interstitial fibrosis in an adenine-induced tubulointerstitial nephritis mouse model. Histochem Cell Biol. 2009 Apr;131(4):483-90. doi: 10.1007/s00418-009-0557-5.

82. Okabe C, Borges RL, de Almeida DC et al. NF- $\kappa B$ activation mediates crystal translocation and interstitial inflammation in adenine overload nephropathy. Am J Physiol Renal Physiol. 2013 Jul 15;305(2):F155-63. doi: 10.1152/ajprenal.00491.2012.

83. Correa-Costa M, Braga TT, Felizardo RJ et al. Macrophage Trafficking as Key Mediator of Adenine-Induced Kidney Injury. Mediators Inflamm. 2014;2014:291024.

84. Mattila JT, Ojo OO, Kepka-Lenhart D et al. Microenvironments in tuberculous granulomas are delineated by distinct populations of macrophage subsets and expression of nitric oxide synthase and arginase isoforms. J Immunol. 2013 Jul 15;191(2):773-84. doi: 10.4049/jimmunol.1300113.

85. Jablonski KA, Amici SA, Webb LM et al. Novel Markers to Delineate Murine M1 and M2 Macrophages. PLoS One. 2015 Dec 23;10(12):e0145342.

86. Fischer HG1, Dörfler R, Schade B, Hadding U. Differential CD86/B7-2 expression and cytokine secretion induced by Toxoplasma gondii in macrophages from resistant or susceptible BALB H-2 congenic mice. Int Immunol. 1999 Mar;11(3):341-9.

87. Nicod LP, Isler P. Alveolar macrophages in sarcoidosis coexpress high levels of CD86 (B7.2), CD40, and CD30L. Am J Respir Cell Mol Biol. 1997 Jul;17(1):91-6. 
88. Rutkowski R, Moniuszko T, Stasiak-Barmuta A et al. CD80 and CD86 Expression on LPSStimulated Monocytes and the Effect of CD80 and CD86 Blockade on IL-4 and IFN- $\gamma$ Production in Nonatopic Bronchial Asthma. Arch Immunol Ther Exp (Warsz). 2003;51(6):421-8.

89. Röszer T. Understanding the Mysterious M2 Macrophage through Activation Markers and Effector Mechanisms. Mediators of Inflammation. 2015; Article ID 816460.

90. Kaku Y, Imaoka H, Morimatsu Y et al. M2 macrophage marker CD163, CD204 and CD206 expression on alveolar macrophages in the lung of patients with chronic obstructive pulmonary. European Respiratory Journal Sep 2014, 44 (Suppl 58) P1481.

91. Pecoits-Filho R, Lindholm B, Axelsson J, Stenvinkel P. Update on interleukin-6 and its role in chronic renal failure. Nephrol Dial Transplant. 2003; 18: 1042-1045.

92. Lee BT, Ahmed FA, Hamm LL, et al. Association of C-reactive protein, tumor necrosis factor-alpha, and interleukin-6 with chronic kidney disease. BMC Nephrology. 2015;16:77.

93. Li C, Ding XY, Xiang DM et al. Enhanced M1 and Impaired M2 Macrophage Polarization and Reduced Mitochondrial Biogenesis via Inhibition of AMP Kinase in Chronic Kidney Disease. Cell Physiol Biochem. 2015;36(1):358-72.

94. Burns WC, Kantharidis P, Thomas MC. The role of tubular epithelial-mesenchymal transition in progressive kidney disease. Cells Tissues Organs. 2007;185(1-3):222-31.

95. Dong D, Cai GY, Ning YC. Alleviation of senescence and epithelial-mesenchymal transition in aging kidney by short-term caloric restriction and caloric restriction mimetics via modulation of AMPK/mTOR signaling. Oncotarget. 2017 Mar 7;8(10):16109-16121. doi: 10.18632/oncotarget.14884.

96. Satriano J, Sharma K, Blantz RC, Deng A. Induction of AMPK activity corrects early pathophysiological alterations in the subtotal nephrectomy model of chronic kidney disease. Am J Physiol Renal Physiol. 2013; 305: F727-F733. doi:10.1152/ajprenal.00293.2013

97. Tikoo K, Sharma E, Amara VR, Pamulapati H, Dhawale VS. Metformin Improves Metabolic Memory in High Fat Diet (HFD)-induced Renal Dysfunction. The Journal of Biological Chemistry. 2016; 291(42):21848-21856.

98. Nikolic-Paterson DJ. CD4 + T cells: a potential player in renal fibrosis. Kidney Int. 2010 Aug;78(4):333-5.

99. Tapmeier TT, Fearn A, Brown K et al. Pivotal role of CD4 $+\mathrm{T}$ cells in renal fibrosis following ureteric obstruction. Kidney Int. 2010 Aug;78(4):351-62.

100. Thakur S, Viswanadhapalli S, Kopp JB et al. Activation of AMP-Activated Protein Kinase Prevents TGF- $\beta 1$-Induced Epithelial-Mesenchymal Transition and Myofibroblast Activation. Am J Pathol. 2015 Aug;185(8):2168-80.

101. Andrzejewski S, Gravel S-P, Pollak M, St-Pierre J. Metformin directly acts on mitochondria to alter cellular bioenergetics. Cancer \& Metabolism. 2014;2:12. 
102. Kang HM, Ahn SH1, Choi P et al. Defective fatty acid oxidation in renal tubular epithelial cells has a key role in kidney fibrosis development. Nat Med. 2015 Jan;21(1):37-46.

103. Grisouard J, Timper K, Radimerski TM, et al. Mechanisms of metformin action on glucose transport and metabolism in human adipocytes. Biochem Pharmacol. 2010 Dec $1 ; 80(11): 1736-45$.

104. Lee JO, Lee SK, Kim JH, et al. Metformin Regulates Glucose Transporter 4 (GLUT4) Translocation through AMP-activated Protein Kinase (AMPK)-mediated Cbl/CAP Signaling in 3T3-L1 Preadipocyte Cells. J Biol Chem. 2012 Dec 28;287(53):44121-9. 